Supporting Information

\title{
Monodisperse and Water-Soluble Quantum Dots for SWIR Imaging via Carboxylic Acid Copolymer Ligands
}

Daniel M. Montana, ${ }^{1}$ Michel Nasilowski, ${ }^{2}$ Whitney R. Hess, ${ }^{2}$ Mari Saif, ${ }^{2}$ Jessica A. Carr, ${ }^{2}$ Lea Nienhaus ${ }^{2,3,{ }^{*}}$ and Moungi G. Bawendi ${ }^{2, *}$

${ }^{1}$ Department of Materials Science and Engineering, ${ }^{2}$ Department of Chemistry, Massachusetts Institute of Technology, Cambridge, Massachusetts 02139, USA, ${ }^{3}$ Department of Chemistry and Biochemistry, Florida State University, Tallahassee, FL, 32306, USA

*corresponding Author: nienhaus@chem.fsu.edu and mgb@mit.edu 


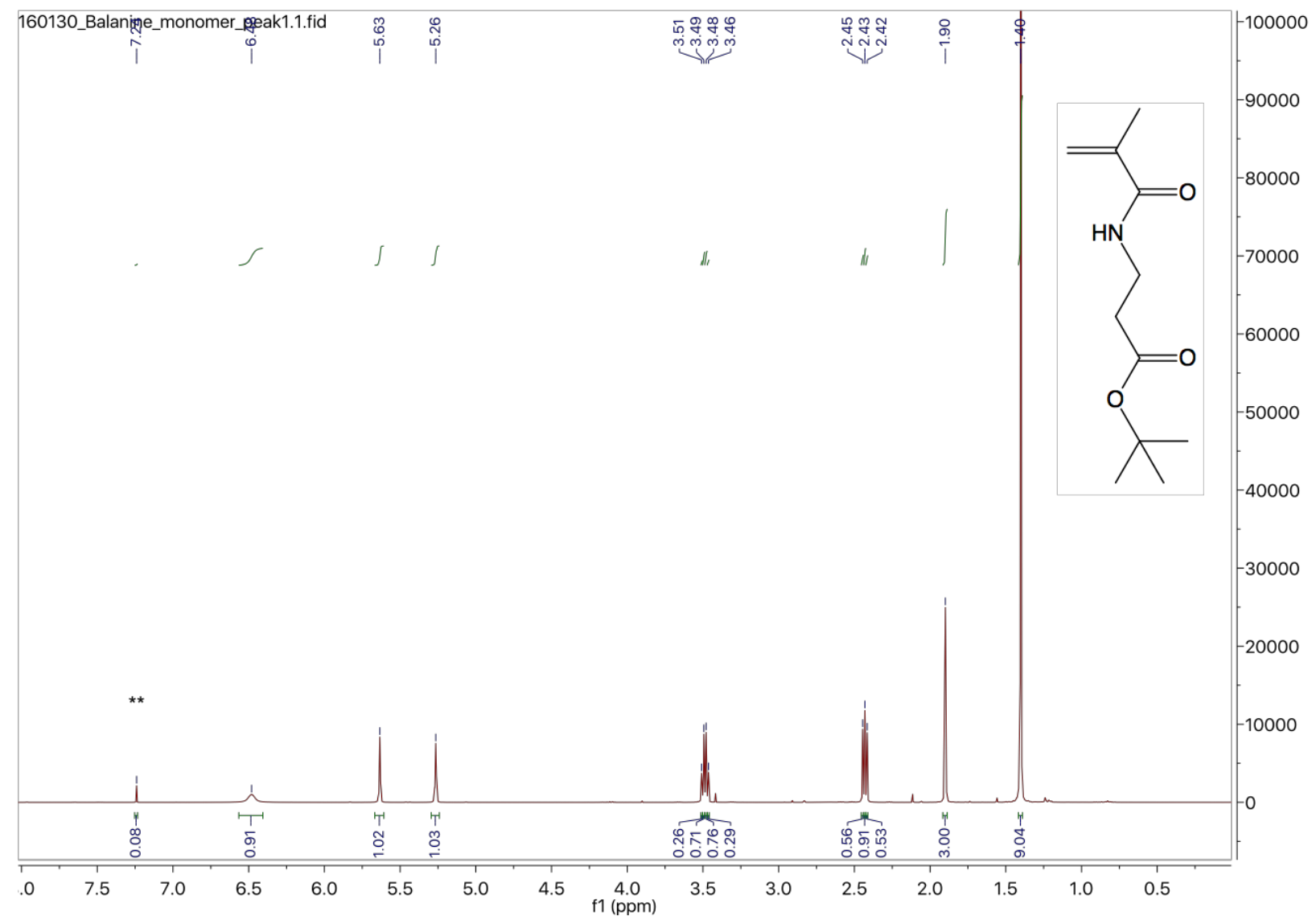

Figure S1: ${ }^{1} \mathrm{H}$ NMR of Monomer 1 in $\mathrm{CDCl}_{3}$. Peak marked ** corresponds to residual $\mathrm{CHCl}_{3}$. Integrals were calibrated relative to peak at $1.90 \mathrm{ppm}$ (set to 3.0 ) 


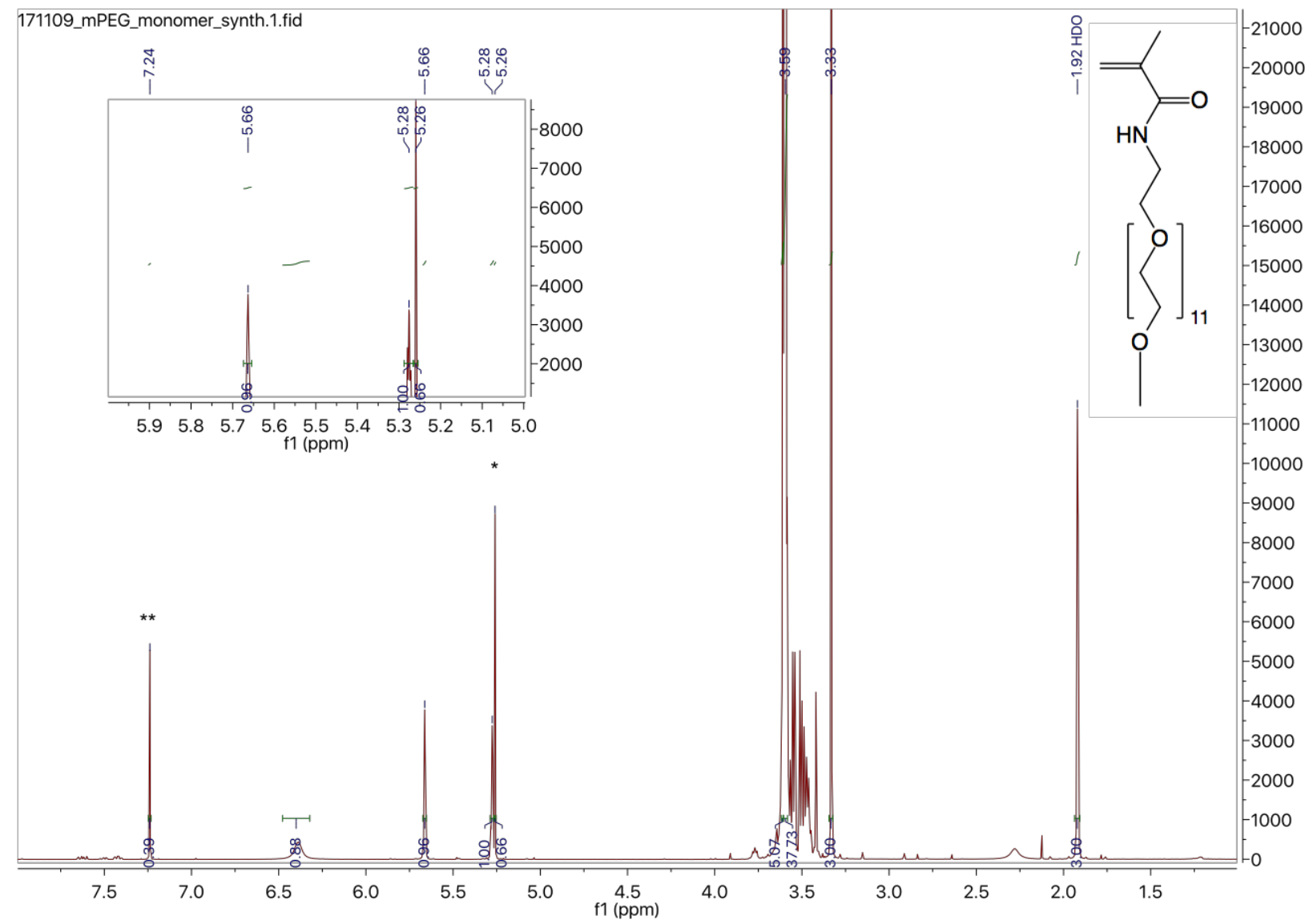

Figure S2: ${ }^{1} \mathrm{H}$ NMR of Monomer 2 in $\mathrm{CDCl}_{3}$. Peak marked ** corresponds to residual $\mathrm{CHCl}_{3}$ and peak marked * corresponds to residual $\mathrm{CH}_{2} \mathrm{Cl}_{2}$. Inset shows further detail of vinyl region. Integrals were calibrated relative to peak at $3.33 \mathrm{ppm}$ (set to 3.0) 


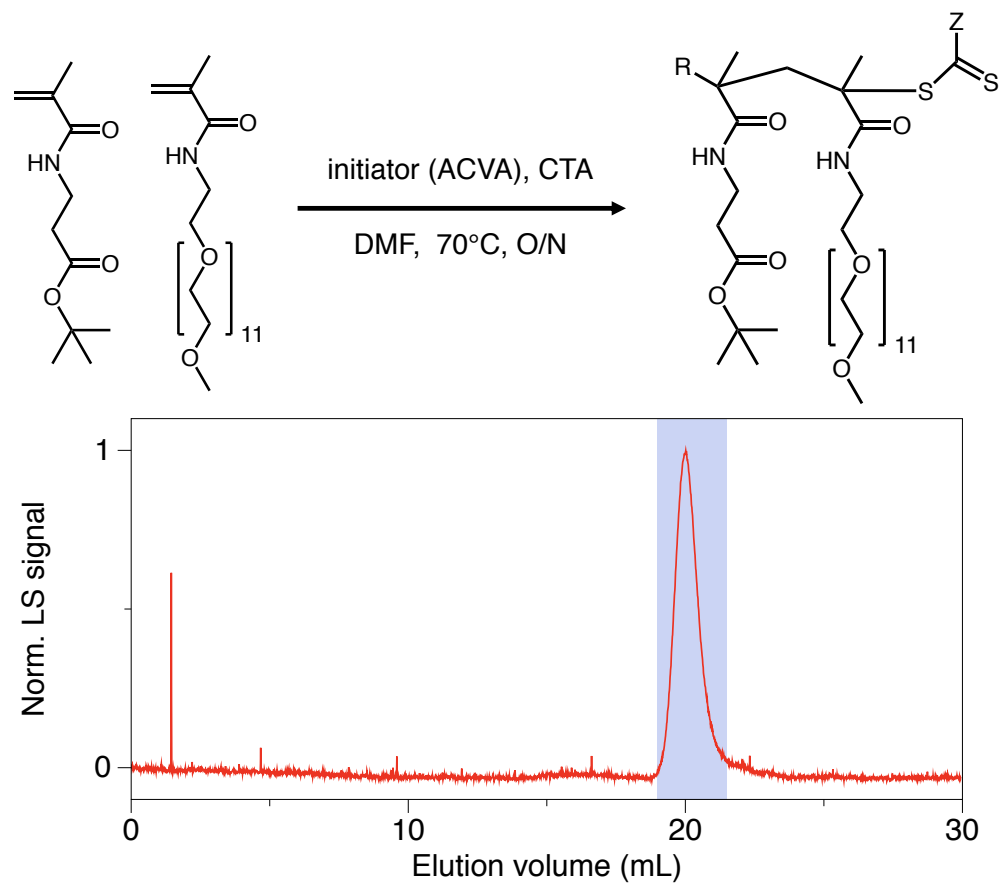

Figure S3: PAL is a random copolymer with a 1:1 molar ratio of monomer 1 and monomer 2 . The reaction is initiated with ACVA and controlled by 4-cyano-4-(thiobenzoylthio)pentanoic acid, with monomer conversion of $80-90 \%$. GPC analysis shows a narrow polymer peak (highlighted) with a $\mathrm{PDI}<1.15$ and estimated $\mathrm{MW} \sim 17 \mathrm{kDa}$ 

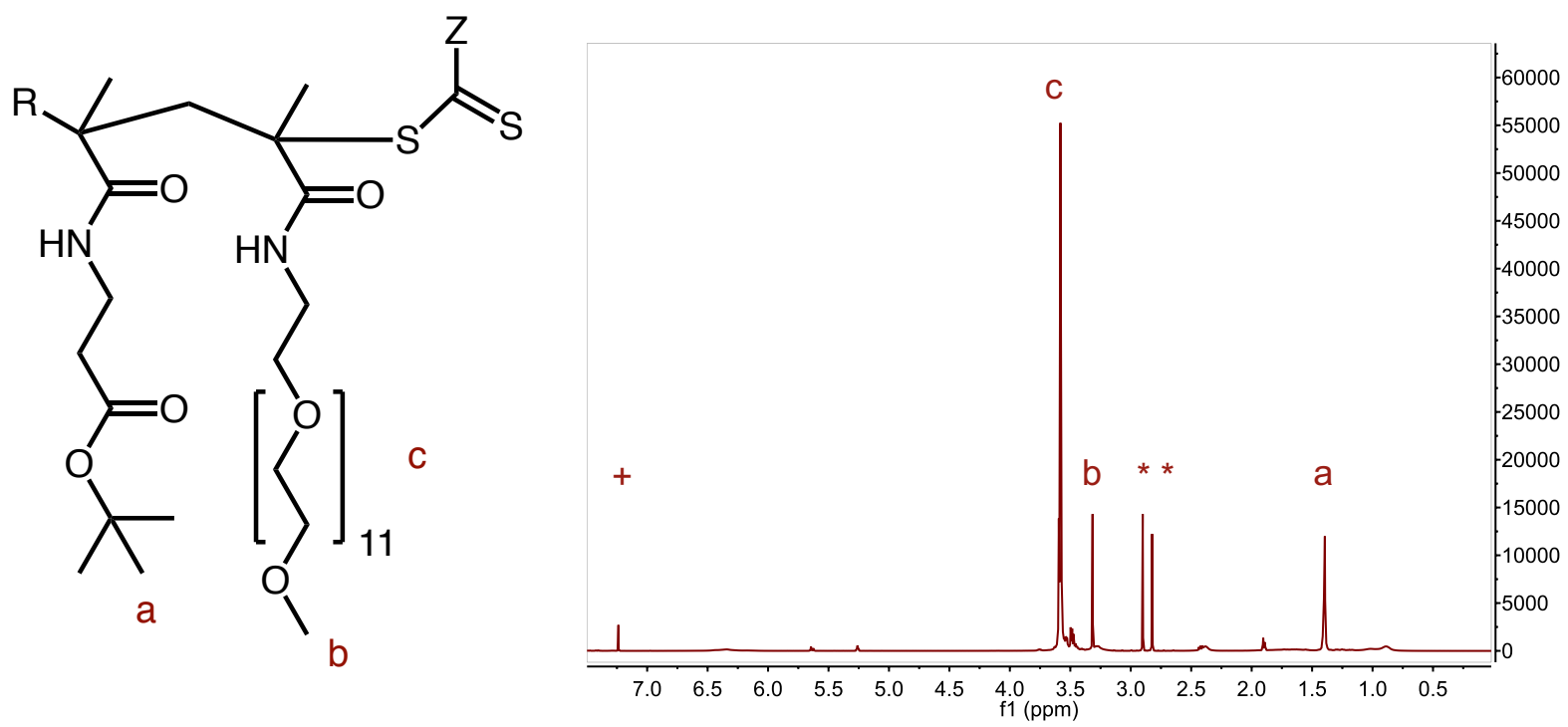

$1: 1\left[\mathrm{CH}_{2} \mathrm{Cl}_{2}\right]:\left[\mathrm{CF}_{3} \mathrm{COOH}\right]$

$\mathrm{RT}, 2 \mathrm{~h}$
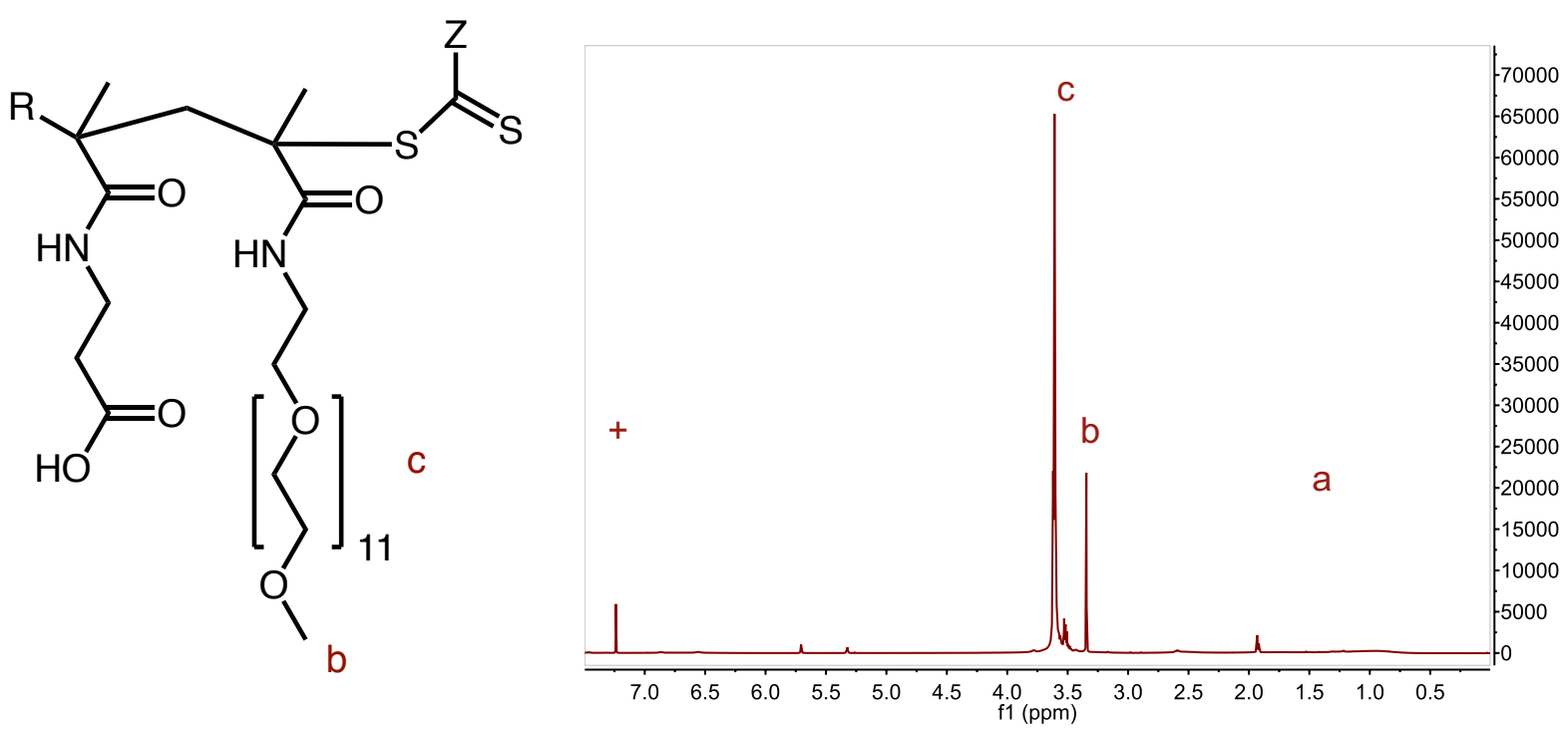

Figure S4: PAL deprotection in dichloromethane and trifluoroacetic acid is $99 \%$ efficient by ${ }^{1} \mathrm{H}$ NMR. The peak corresponding to the tert-butyl group (a) disappears nearly entirely after deprotection and drying. The terminal methyl group on PEG (b) is used as an internal reference for peak integration. (c) corresponds to protons on PEG chains. The peaks marked with * correspond to residual DMF from polymerization, and the peak marked + corresponds to $\mathrm{CHCl}_{3}$ in the NMR solvent $\left(\mathrm{CDCl}_{3}\right)$. 


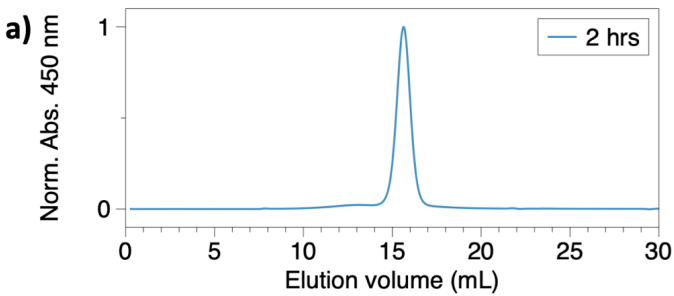

b)
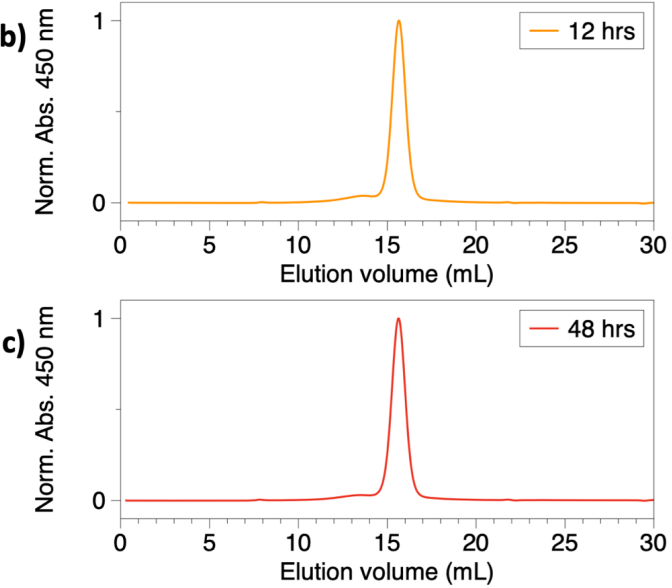

d)

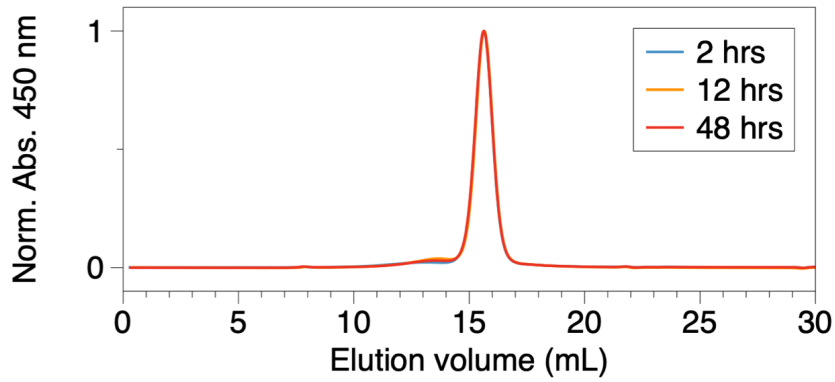

Figure S5: Time evolution of ligand exchange. The GFC traces of PAL ligand exchange experiments with $\mathrm{PbS} / \mathrm{CdS}$ QDs after ligand exchange for 2 hours (a), 12 hours (b), and 48 hours (c) are nearly identical when overlaid (d) suggesting that ligand exchange is complete after only a short exchange. 

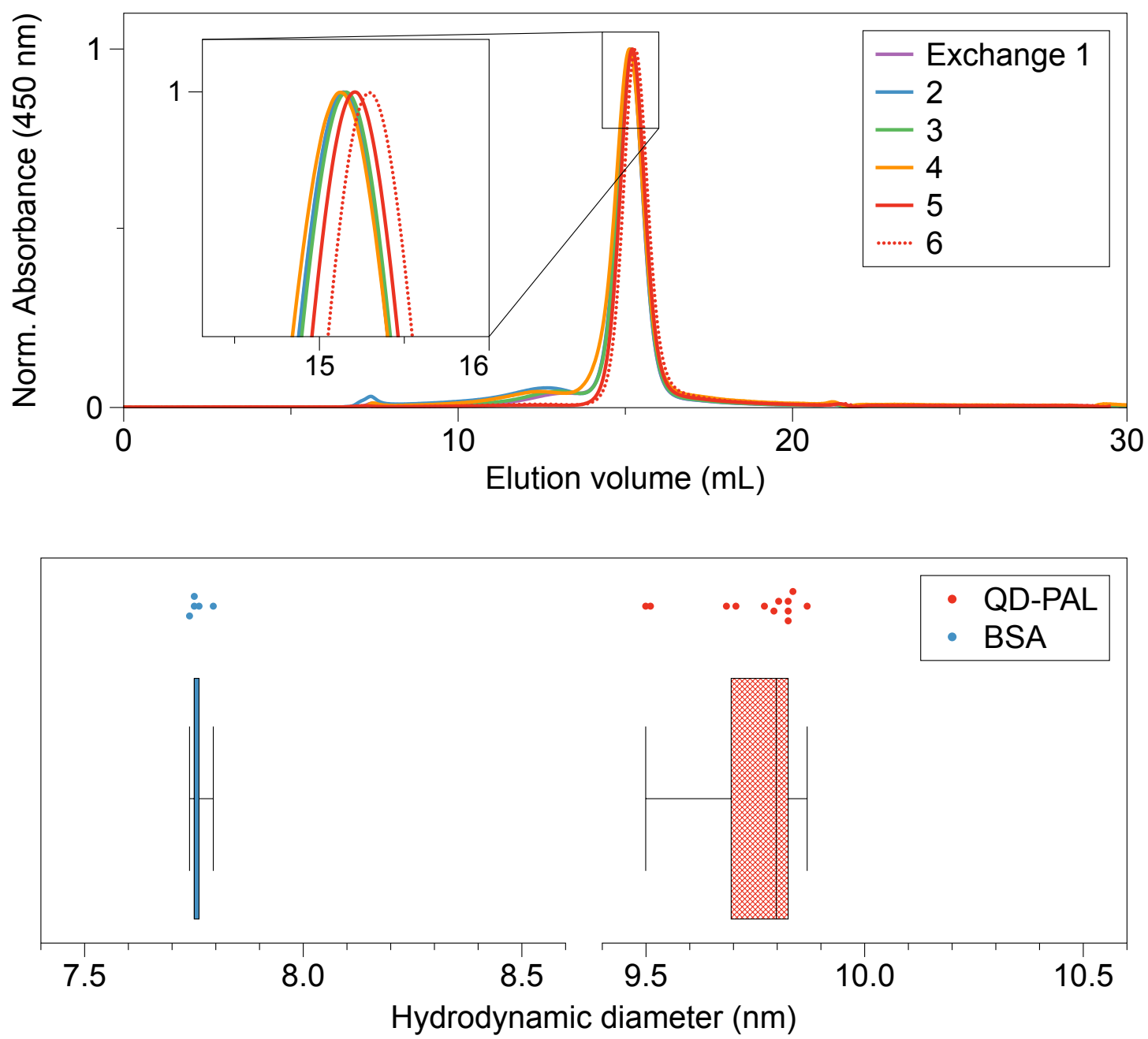

Figure S6: GFC analysis of six exchange experiments of $\mathrm{PbS} / \mathrm{CdS}$ QDs with PAL shows very similar particle distributions (top). The inset shows closer detail on the elution peak and slight differences between some experiments are discernible. On the bottom, we show a statistical analysis of the elution peak for twelve such exchange experiments (red). The whiskers show the extremes of the distribution and each experiment's $H_{d}$ is plotted above the box. The average calculated $\mathrm{H}_{\mathrm{d}}$ for our QD-PAL probes is $9.75 \mathrm{~nm}$ with a standard deviation of $0.12 \mathrm{~nm}$. For comparison, six samples of bovine serum albumin -a monodisperse macromolecule standard- (BSA, avg. $\mathrm{H}_{\mathrm{d}} 7.76 \mathrm{~nm}$, blue) were analyzed on the same GFC instrument, giving a standard deviation of $0.018 \mathrm{~nm}$ in $\mathrm{H}_{\mathrm{d}}$. 